\title{
Electrostatic pair creation and recombination in quantum plasmas
}

\author{
M. Marklund, ${ }^{1}$ B. Eliasson, ${ }^{2}$ P. K. Shukla, ${ }^{1,2}$ L. Stenflo, ${ }^{1}$ M. E. Dieckmann, ${ }^{2}$ and M. Parviainen ${ }^{2}$ \\ ${ }^{1}$ Centre for Nonlinear Physics, Department of Physics, Umeå University, SE-901 87 Umeå, Sweden \\ ${ }^{2}$ Institut für Theoretische Physik IV, Ruhr-Universität Bochum, D-44780 Bochum, Germany
}

The collective production of electron-positron pairs by electrostatic waves in quantum plasmas is investigated. In particular, a semi-classical governing set of equation for a self-consistent treatment of pair creation by the Schwinger mechanism in a quantum plasma is derived.

\section{INTRODUCTION}

In quantum field theory, and quantum electrodynamics (QED) in particular, the vacuum is no longer trivial, but may under certain circumstances act as a medium. Effects such as elastic light-by-light scattering as photon splitting are well known examples of the influence of the nonlinear quantum vacuum [1]. The concept of pair creation is of great interest both from a principal point of view, as well as for near-future applications. In particular, the production of pairs in an intrinsically nonperturbative process, and as such poses new requirements for its theoretical treatment. Lately, there has been much interest in pair creation due to nontrivial generalizations of the constant electric field case (see e.g. $[2,3,4,[5,6,7,8,9]$ and references therein), as considered by Schwinger for quantum electrodynamics [10]. The next generation laser systems, such as the X-ray free electron laser, will be able to produce pairs, and this necessitates the study of collective and self-consistent pair production effects. Thus, in this spirit, the effects of pair production on circularly polarized light was considered in Refs. 15] and [16], and it was found that electromagnetic degrees of freedom were dissipated into pairs in a selfconsistent manner. However, it is not unreasonable to assume that within laser-plasma and astrophysical systems, strong electrostatic fields may be built up, thus posing a new problem concerning pair creation.

In this paper we will study the effects of electrostatic pair creation in a three component plasma, consisting of ions, electrons, and positrons. The quantum properties of electrons and positrons are partially included by using a semi-classical approximation of the many-body Schrödinger system. The effect of the latter is to introduce higher order dispersion in the electron/positron momentum equations. The combined effect of pair creation and quantum plasmas can thus be studied using and effective semi-classical theory in terms of macroscopic fluid variables.

\section{THEORY}

For fields varying slowly compared to the Compton frequency $\omega_{e}=m_{e} c^{2} / \hbar$, where $m_{e}$ is the electron rest mass, $c$ is the velocity of light in vacuum, and $\hbar$ is the Planck constant divided by $2 \pi$, the pair creation rate per unit phase space volume given by $q(t, \vec{r}, \vec{p})=q_{0}(t, \vec{r}) F(\vec{p})$, where 10, 11, 12, 13]

$$
q_{0}=\frac{c}{(2 \pi)^{3} \lambda^{4}} \frac{|\vec{E}|^{2}}{E_{\text {crit }}^{2}} \exp \left(-\pi \frac{E_{\text {crit }}}{|\vec{E}|}\right),
$$

Here $\vec{p}$ is the electron/positron microscopic momentum, $\vec{E}$ is the electric field strength, $e$ is the magnitude of the electron charge, $E_{\text {crit }}=m_{e}^{2} c^{3} / e \hbar \sim 10^{16} \mathrm{~V} \mathrm{~cm}^{-1}$ is the Schwinger critical field, $\lambda=\hbar / m_{e} c$ is the Compton wavelength, $m_{e}$ is the electron rest mass, $c$ is the speed of light in vacuum, $\hbar$ in Planck's constant divided by $2 \pi$, and the momentum distribution function is normalized according to $\int F d^{3} p=1$. produced at rest, thus the delta function in momentum space, and neglected the small correction due to the electron/positron momentum orthogonal to the electric field. We note that the expression (11) should be used with caution, as pointed out in Ref. 6], as the electrons/positrons does not experience the local field. This can be partially remedied by averaging the field over a suitable spatial volume. Here we will at present consider the local field approximation, keeping in mind the above. Apart from the pair creation rate, there will also be a pair recombination, or annihilation, rate given by $\nu_{e}=\sigma v_{c} n_{p}\left(\nu_{p}=\sigma v_{c} n_{e}\right)$ for the electrons (positrons), where $\sigma$ is the cross section, $v_{c}$ is a characteristic velocity of the recombination, and $n_{e}\left(n_{p}\right)$ is the electron (positron) number density.

For electrostatic oscillations, the electric field is determined by Poisson's equation

$$
\vec{\nabla} \cdot \vec{E}=\left(-e n_{e}+e n_{p}+Z_{i} e n_{i}+\rho_{\mathrm{pol}}\right) / \epsilon_{0} .
$$

where $n_{e}\left(n_{p}\right)$ is the electron (positron) number density in the laboratory frame, $n_{i}$ is the ion number density, $Z_{i} e$ is the ion charge density and $\rho_{\text {pol }}$ is a vacuum polarization charge density. Ampère's law gives

$$
\partial_{t} \vec{E}=\frac{1}{\epsilon_{0}}\left(\frac{e n_{e} \vec{p}_{e}}{\gamma_{e} m_{e}}-\frac{e n_{p} \vec{p}_{p}}{\gamma_{p} m_{p}}-\frac{Z_{i} e n_{i} p_{i}}{\gamma_{i} m_{i}}-\vec{j}_{\mathrm{pol}}\right)
$$

where the polarization current is $\vec{j}_{\text {pol }}=\vec{E} q_{0} /|\vec{E}|^{2}$ and the vacuum polarization charge density is defined via the continuity equation $\partial_{t} \rho_{\text {pol }}+\vec{\nabla} \cdot \overrightarrow{j_{\text {pol }}}=0$. The particular choice of $\vec{j}_{\text {pol }}$ gives overall energy conservation to the system where the energy of newly created pairs is compensated by a decrease of the electrostatic energy. The fluid equation for the cold electrons, positrons, and ions 
are governed by 14,16 .

$$
\begin{aligned}
& \partial_{t} n_{e}+\vec{\nabla} \cdot\left(\frac{n_{e} \vec{p}_{e}}{\gamma_{e} m_{e}}\right)=q_{0}-\sigma v_{c} n_{e} n_{p}, \\
& \partial_{t} n_{p}+\vec{\nabla} \cdot\left(\frac{n_{p} \vec{p}_{p}}{\gamma_{p} m_{p}}\right)=q_{0}-\sigma v_{c} n_{e} n_{p}, \\
& \partial_{t} n_{i}+\vec{\nabla} \cdot\left(\frac{n_{i} \vec{p}_{i}}{\gamma_{i} m_{i}}\right)=0,
\end{aligned}
$$

and

$$
\begin{gathered}
\left(\partial_{t}+\frac{\vec{p}_{e}}{\gamma_{e} m_{e}} \cdot \vec{\nabla}\right) \vec{p}_{e}=-e \vec{E}+\frac{\hbar^{2}}{2 m_{e} \gamma_{e}} \vec{\nabla} U_{\mathrm{B} e} \\
+\frac{q_{0}}{n_{e}}\left(\vec{P}_{e}-\vec{p}_{e}\right)-\sigma v_{c} n_{p}\left(\vec{p}_{e}-\vec{p}_{p}\right), \\
\left(\partial_{t}+\frac{\vec{p}_{p}}{\gamma_{p} m_{p}} \cdot \vec{\nabla}\right) \vec{p}_{p}=e \vec{E}+\frac{\hbar^{2}}{2 m_{p} \gamma_{p}} \vec{\nabla} U_{\mathrm{B} p} \\
+\frac{q_{0}}{n_{p}}\left(\vec{P}_{p}-\vec{p}_{p}\right)-\sigma v_{c} n_{e}\left(\vec{p}_{p}-\vec{p}_{e}\right), \\
\left(\partial_{t}+\frac{\vec{p}_{i}}{\gamma_{i} m_{i}} \cdot \vec{\nabla}\right) \vec{p}_{i}=Z_{i} e \vec{E},
\end{gathered}
$$

valid for length scales larger than $v_{F} / \omega_{\text {pe }}, v_{F}$ being the Fermi velocity and $\omega_{\mathrm{p} e}=\left(n_{0} e^{2} / \epsilon_{0} m_{e}\right)^{1 / 2}$ is the electron plasma frequency for some typical electron density $n_{0}$. Here we have added the recombination term for the sake of generality, and the index $e(p)$ denotes the electrons (positrons), $i$ denotes the ions, $n_{e, p, i}$ is the number density, $\vec{p}_{e, p, i}$ is the fluid momentum, $-e(+e)$ is the electron (positron) charge, $Z_{i} e$ is the ion charge, and $\gamma_{e, p, i}=\left(1+p_{e, p, i}^{2} / m_{e, p, i}^{2} c^{2}\right)^{1 / 2}$ is the relativistic gamma factor. Moreover, the generalized relativistic Bohm potential is defined according to (neglecting the spin of the particles)

$$
\begin{gathered}
U_{\mathrm{B} e, p}=\left(n_{e, p} / \gamma_{e, p}\right)^{-1 / 2} \nabla^{2}\left(n_{e, p} / \gamma_{e, p}\right)^{1 / 2} \\
-\left(n_{e, p} / \gamma_{e, p} c^{2}\right)^{-1 / 2} \partial_{t}^{2}\left(n_{e, p} / \gamma_{e, p}\right)^{1 / 2}
\end{gathered}
$$

obtained using a Madelung representation for the Klein-Gordon equation, and we have defined $\vec{P}_{e, p}=$ $\int \vec{p}_{e, p} F d^{3} p$. Equation (2a)-(2h), together with Eq. (3), determines the semi-classical dynamics of the three component plasma where quantum mechanical correction for the electrons and positrons are taken into account.

\section{ONE-DIMENSIONAL MODEL}

We now specialize to a one-dimensional geometry. In addition to the effects discussed in the $3 \mathrm{D}$ model, we will assume that the electron-positron pairs are created at some distance from each other so that the energy of the particles are compensated by a decrease of the electric field energy. The Poisson equation is on the form

$$
\partial_{x} E=\frac{e}{\epsilon_{0}}\left(n_{p}-n_{e}-Z_{i} n_{i}\right)
$$

where ions are taken to be immobile so that $Z_{i} n_{i}=n_{0}$. We assume that the the electron and positron continuity equations, respectively, are on the form

$$
\partial_{t} n_{e}+\partial_{x}\left(\frac{n_{e} p_{e}}{\gamma_{e} m_{e}}\right)=Q_{e}
$$

and

$$
\partial_{t} n_{p}+\partial_{x}\left(\frac{n_{p} p_{p}}{\gamma_{p} m_{p}}\right)=Q_{p}
$$

and the momentum equations

$$
\partial_{t} p_{e}+m_{e} c^{2} \partial_{x} \gamma_{e}=-e E
$$

and

$$
\partial_{t} p_{p}+m_{p} c^{2} \partial_{x} \gamma_{p}=e E
$$

where we have assumed that the cold particles are created with momenta equal to the fluid momenta of the electrons and positrons, respectively, so that the sources do not contribute to the momenta of the particles but only to the particle number densities. In this manner, we do not have heating of the particles and do not need to include pressure or take into account kinetic effects. The source terms are taken to be on the form

$$
Q_{e}=q_{0}+\partial_{x}\left(\lambda \gamma_{e} q_{0} \frac{E_{\text {crit }}}{E}\right)
$$

and

$$
Q_{p}=q_{0}-\partial_{x}\left(\lambda \gamma_{p} q_{0} \frac{E_{\text {crit }}}{E}\right)
$$

and correspond roughly to that the creation of electrons and positrons at the position $x$ depends on the electric field at a distance $\lambda \gamma_{p} E_{\text {crit }} / E$ and $-\lambda \gamma_{e} E_{\text {crit }} / E$, respectively, from $x$. For a stronger electric field, the the distance of creation decreases, while it increases for larger $\gamma_{j}$. This form on the source term gives an approximate energy conservation law of the form

$$
\begin{gathered}
\frac{d}{d t} \int_{\Omega}\left[m_{e} c^{2}\left(n_{e} \gamma_{e}+n_{p} \gamma_{p}-2 n_{0}\right)+\frac{\epsilon_{0} E^{2}}{2}\right] d x \\
=m c^{2} \int \frac{E_{\mathrm{crit}}}{2 E} q_{0} \lambda \partial_{x}\left(\gamma_{e}^{2}-\gamma_{p}^{2}\right) d x
\end{gathered}
$$

where the right-hand side can be assumed to be small if $\lambda\left|\partial_{x}\right| \ll 1$, and where we have assumed that all fields and velocities vanish at $|x|=\infty$. The conservation law was obtained by using Ampères law

$$
\partial_{t} E=\frac{e}{\epsilon_{0}}\left[\frac{n_{e} p_{e}}{\gamma_{e} m_{e}}-\frac{n_{p} p_{p}}{\gamma_{p} m_{p}}+\left(\gamma_{e}+\gamma_{p}\right) \lambda \frac{E_{\mathrm{crit}}}{E} q_{0}\right],
$$

which is derived by differentiating the Poisson equation by time and using the continuity equations for the particles. 


\section{THE NORMALIZED SYSTEM OF EQUATIONS}

We may normalize the system of equations (2) by introducing the Compton wave length $\lambda$ and the time scale $\tau=\hbar / m_{e} c^{2}$, normalizing the electric field by $E_{\text {crit }}$, normalizing all momenta by $m_{e} c$ (with $m_{p}=m_{e}$ ), and normalizing the number densities by some preferred density $n_{0}$. Assuming stationary ions, we then obtain the normalized and dimensionless system

$$
\begin{gathered}
\vec{\nabla} \cdot \vec{E}=\omega_{\mathrm{p} e}^{2}\left(1-n_{e}+n_{p}\right), \\
\partial_{t} n_{e}+\vec{\nabla} \cdot\left(\frac{n_{e} \vec{p}_{e}}{\gamma_{e}}\right)=q_{0}-a n_{e} n_{p}, \\
\partial_{t} n_{p}+\vec{\nabla} \cdot\left(\frac{n_{p} \vec{p}_{p}}{\gamma_{p}}\right)=q_{0}-a n_{e} n_{p},
\end{gathered}
$$

and

$$
\begin{gathered}
\left(\partial_{t}+\frac{\vec{p}_{e}}{\gamma_{e}} \cdot \vec{\nabla}\right) \vec{p}_{e}=-\vec{E}+\frac{1}{2} \vec{\nabla} U_{\mathrm{B} e}+\frac{q_{0}}{n_{e}}\left(\vec{P}_{e}-\vec{p}_{e}\right) \\
\quad-a n_{p}\left(\vec{p}_{e}-\vec{p}_{p}\right), \\
\left(\partial_{t}+\frac{\vec{p}_{p}}{\gamma_{p}} \cdot \vec{\nabla}\right) \vec{p}_{p}=\vec{E}+\frac{1}{2} \vec{\nabla} U_{\mathrm{B} p}+\frac{q_{0}}{n_{p}}\left(\vec{P}_{p}-\vec{p}_{p}\right) \\
\quad-a n_{e}\left(\vec{p}_{p}-\vec{p}_{e}\right),
\end{gathered}
$$

where now

$$
q_{0}=\left(E^{2} / N_{0}\right) \exp \left(-\pi|E|^{-1}\right)
$$

and we have used the stationary ion background density as the normalization density $n_{0}, N_{0}=n_{0} h^{3} / m_{e}^{3} c^{3}$, the electron plasma frequency is normalized by $1 / \tau$ such that $\widetilde{\omega}_{\mathrm{p} e}=\left(2 \alpha N_{0}\right)^{1 / 2} / 2 \pi, \alpha=e^{2} / 4 \pi \epsilon_{0} \hbar c \approx 1 / 137$ is the fine structure constant, and we have denoted $a=$ $N_{0}\left(\sigma / \lambda^{2}\right)\left(v_{c} / c\right)$.

\section{NUMERICAL ANALYSIS OF THE ONE-DIMENSIONAL SYSTEM}

The one-dimensional system of equations (4)-(8) are normalized so that

$$
\begin{gathered}
\partial_{x} E=\widetilde{\omega}_{\mathrm{p} e}^{2}\left(1-n_{e}+n_{p}\right) \\
\partial_{t} n_{e}+\partial_{x}\left(\frac{n_{e} p_{e}}{\gamma_{e}}\right)=q_{0}+\partial_{x}\left(\frac{\gamma_{e} q_{0}}{E}\right), \\
\partial_{t} n_{p}+\partial_{x}\left(\frac{n_{p} p_{p}}{\gamma_{p}}\right)=q_{0}-\partial_{x}\left(\frac{\gamma_{e} q_{0}}{E}\right), \\
\left(\partial_{t}+\frac{p_{e}}{\gamma_{e}} \partial_{x}\right) p_{e}=-E \\
\left(\partial_{t}+\frac{p_{p}}{\gamma_{p}} \partial_{x}\right) p_{p}=E .
\end{gathered}
$$

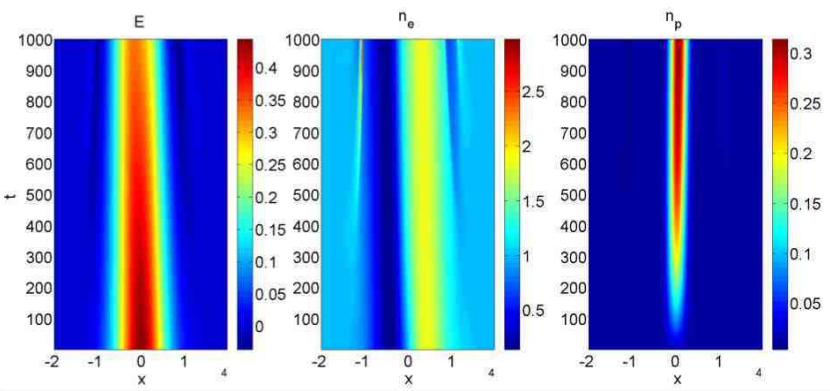

FIG. 1: The electrostatic field (left panel) electron density (middle panel) and positron density (right panel) as a function of space $x$ and time $t$. We used $N_{0}=0.2$ and the initial conditions $n_{e}=1.01+2(x / L) \exp \left(-x^{2} / L^{2}\right)$ with $L=6000$, and $n_{p}=0.01$, for the electron and positron number densities, respectively.

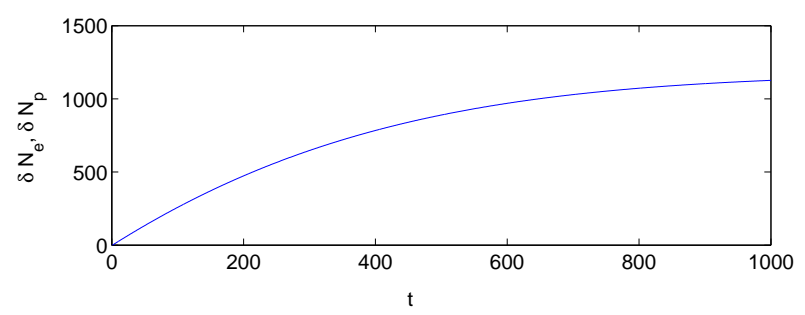

FIG. 2: The total number of created electron-positron pairs, $\delta N_{p}=\delta N_{e}=N_{e}-N_{e, t=0}$, where $N_{e}=\int_{\omega} n_{e} d x$, as a function of time. We see a decrease in the pair creation rate, correlated with a decrease of the amplitude of the electrostatic field seen in Fig. 1.

and are solved numerically. The results are displayed in Figs. (1)-(3). Initially, the electron density is perturbed locally on the form $n_{e}=1.01+2(x / L) \exp \left(-x^{2} / L^{2}\right)$ with $L=6000$, and for the positrons we take a small non-zero component $n_{p}=0.01$. All particle species are assumed initially to be in rest, i.e., $p_{e}=p_{p}=0$ at $t=0$. We have assumed a dense plasma so that $N_{0}=0.2$. Ini-

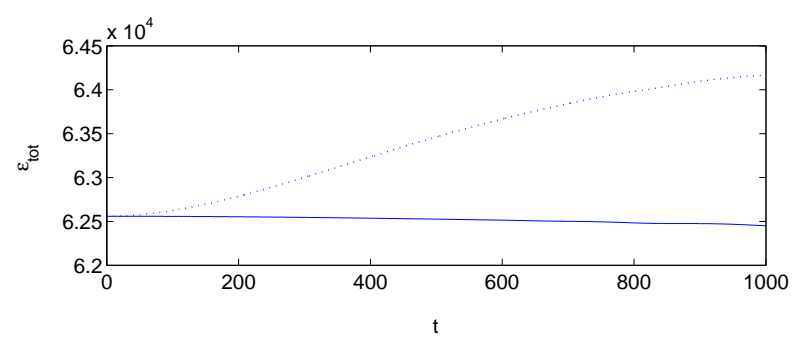

FIG. 3: The total energy $\mathcal{E}_{\text {tot }}=\int_{\Omega}\left(n_{e} \gamma_{e}+n_{p} \gamma_{p}+E^{2} / 2 \widetilde{\omega}_{p}^{2}\right) d x$ as a function of time (solid curve). The dotted curve displays the same integral for the case when the correction for the spatial displacement of the created pairs, the last terms in the right-hand sides of Eqs. 15b and (15c), are neglected. In the latter case, the creation of electron-positron pairs lead to an increase of the total energy in the system. 
tially, the maximum electric field is approximately half the Schwinger field, $E_{\max } \simeq 0.5$, and we see in the righthand panel of Fig. 1 that electron-positron pairs are created so that the particle density of the positrons are increased in a small region around $x=0$ where the electric field amplitude has its maximum. Due to the ultra-strong electric field, the electrons are also accelerated so as to neutralize the plasma, and hence the electric field strength decreases with time. We see in Fig. 2, that the pair creation rate is largest initially, when the electric field is strongest, and decreases at later times. Both the electrons and positrons are accelerated to ultra-relativistic speeds with gamma factors of the order 100-500, and the dynamics is essentially a balance between the kinetic energy of the particles and the potential energy stored in the electric field. We consider the energy balance in Fig. 3, where we have plotted the time evolution of the sum of the total relativistic particle energies and the electrostatic energy. We see that this energy is approximately conserved (solid line) when the the full expressions for the pair creation in the continuity equations (15b)-15c) are used. The last terms in the continuity equation account approximately for that the electron and positron is created at some spatial difference from each other so that the total energy of the newly created pair is compensated by a decrease of the electric field and hence of the electrostatic energy. If the last terms in the right-hand sides of the continuity equations are neglected, meaning that the pairs are created at the same point in space, then there is a visible increase of the total energy when the pairs are created (the dotted curve in Fig. 3). However, the energy loss to the pairs is relatively small compared to the total free energy of the system, stored initially in the electrostatic field.

In summary, we have presented a dynamical and selfconsistent model for the electron-positron pair creation by a strong electrostatic field in a dense plasma. The electrostatic field is excited self-consistently by largeamplitude electron waves, which gives rise to electric fields that are comparable with Schwinger's critical field. We have derived an approximately energy-conserving one-dimensional model that takes into account the decrease of the electrostatic energy as electron-positron pairs are created. The model presented here constitutes a first step of understanding the dynamics of largeamplitude electrostatic waves that are strong enough to create electron-positron pairs. A future model should be derived from first principles of quantum electrodynamics, to take into account the distribution of the created electron positron pairs in momentum space and the selfconsistent energy conservation of the system.

\section{Acknowledgment}

This work was partially supported by the Swedish Research Council and by the DFG through the SFB 591.
[1] M. Marklund and P. K. Shukla, Rev. Mod. Phys. 78, No. 2 , in press (2006).

[2] E. Brezin and C. Itzykson, Phys. Rev. D 2, 1191 (1970).

[3] R. Alkofer, M. B. Hecht, C. D. Roberts, S. M. Schmidt, and D. V. Vinnik, Phys. Rev. Lett. 87, 193902 (2001).

[4] H. Nitta, M. Kh. Khokonov, Y. Nagata, and S. Onuki, Phys. Rev. Lett. 93, 180407 (2004).

[5] D. B. Blaschke, A. V. Prozorkevich, C. D. Roberts, S. M. Schmidt, and S. A. Smolyanski, nucl-th/0511085

[6] H. Gies and K. Klingmüller, Phys. Rev. D 72, 065001 (2005).

[7] H. Gies, J. Sanchez-Guillen, R. A. Vázquez, JHEP 08, 067 (2005).

[8] G. V. Dunne and C. Schubert, Phys. Rev. D 72, 105004 (2005).

[9] H. M. Fried and Y. Gabellini, Phys. Rev. D 73,
011901(R) (2006)

[10] J. Schwinger, Phys. Rev. 82, 664 (1951).

[11] K. Kajantie and T. Matsui, Phys. Lett. B 164, 373 (1985).

[12] G. Gatoff, A. K. Kerman, and T. Matsui, Phys. Rev. D 36,114 (1987).

[13] Y. Kluger, J. M. Eisenberg, and B. Svetitsky, Phys. Rev. Lett. 67, 2427 (1991).

[14] G. Manfredi, in Topics in Kinetic Theory, Eds. T. Passot, C. Sulem, and P.-L. Sulem, (Fields Institute Communications, American Mathematical Society, 2005), quant-ph/0505004

[15] S. S. Bulanov, Phys. Rev. E 69, 036408 (2004).

[16] S. S. Bulanov, A. M. Fedotov, and F. Pegoraro, Phys. Rev. E 71, 016404 (2005). 